\section{PC-016 STABILITY OF COMPOUNDED NIVOLUMAB SOLUTION AFTER PNEUMATIC SYSTEM TRANSPORTATION}

1,2L Camuffo*, ${ }^{3} \mathrm{~F}$ Selmin, ${ }^{5} \mathrm{~F}$ Vasile, 'M Rivano, ${ }^{1} \mathrm{M}$ Piccoli, ' $\mathrm{G}$ Mangoni, 'L Cancanelli, ${ }^{4} \mathrm{M}$ Fazio, ${ }^{3} \mathrm{~F}$ Cilurzo, ${ }^{3} \mathrm{P}$ Minghetti. ${ }^{1}$ University of Milan, School in Hospital Pharmacy, Milan, Italy; ${ }^{2}$ Humanitas Research Hospital, Pharmacy, Rozzano, Italy; ${ }^{3}$ University of Milan, Department of Pharmaceutical Sciences, Milan, Italy; ${ }^{4}$ San Raffaele Hospital, Pharmacy, Milan, Italy; ${ }^{5}$ University of Milan, Department of Chemistry, Milan, Italy

\subsection{6/ejhpharm-2020-eahpconf.63}

Background and importance Pneumatic delivery is not recommended for medicinal products that could undergo physical alteration of the active ingredient, such as protein denaturation (Peak, 2003). A review of the literature reveals that the solution air-liquid interface and number of travel cycles can be risk determining factors for compounded stability of monoclonal antibodies after pneumatic delivery (Vieillard et al, 2012; Vieillard et al, 2013; Vieillard et al, 2014). In our hospital, all compounded monoclonal antibodies are delivered via a pneumatic system to the oncologic day hospital unit from the pharmacy compounding department.

Aim and objectives To investigate the stability of nivolumab compounded solution after pneumatic delivery, and the effect of residual air inside the infusion bag.

Material and methods The following nivolumab samples, diluted to $2.4 \mathrm{mg} / \mathrm{mL}$ in a prefilled $0.9 \%$ sodium chloride polyolefin infusion bag, were prepared: sample nivolumab, not undergoing pneumatic delivery, sample PNA, with residual air, and sample $\mathrm{PN}$, without residual air, both undergoing single travel inside the pneumatic delivery system. On the day of preparation, all samples were analysed for $\mathrm{pH}$, osmolality, turbidimetry, dynamic light scattering (DLS), size exclusion chromatography-high performance liquid chromatography (SECHPLC) and nuclear magnetic resonance (NMR).

Results All samples were clear, without particulate or precipitates, and turbidity free at $350 \mathrm{~nm}$. pH values shifted from 5.77 to 5.92. Osmolality values ranged from 286 and 296 $\mathrm{mOsm} / \mathrm{kg}$. DLS revealed a monodisperse peak at about 11 $\mathrm{nm}$, with similar shape and intensity. SEC-HPLC did not reveal any peak retention time variations, and NMR did not reveal any modifications regarding peak shape or intensity.

Conclusion and relevance No difference in physical or chemical stability was found between compounded nivolumab solutions not undergoing and undergoing single travel inside the pneumatic system. The presence of the air-liquid interface inside the solution bag was not risk determining for solution stability. The pneumatic delivery system at our hospital can be used for delivery of compounded nivolumab solution to the oncologic day hospital.

\section{REFERENCES AND/OR ACKNOWLEDGEMENTS}

No conflict of interest.

\section{PC-017 INCREASING PHARMACY PRODUCTIVITY BY EXPANDING THE ROLE OF THE INTRAVENOUS COMPOUNDING ROBOT IN A COMPREHENSIVE CANCER CENTRE}

${ }^{1} \mathrm{M}$ Capilli ${ }^{*},{ }^{2} \mathrm{M}$ Federici, ${ }^{3} \mathrm{~T}$ Comandone, ${ }^{1} \mathrm{G}$ Maffeis, ${ }^{4} \mathrm{~F}$ Foglio, ${ }^{4} \mathrm{~L}$ Omini, ${ }^{4} \mathrm{~F}$ Enrico. ${ }^{1}$ University of Turin, Drug Science and Technology-School of Specialisation in Hospital Pharmacy, Turin, Italy; ${ }^{2}$ Loccioni, Angeli Di Rosora, Ancona, Italy; ${ }^{3}$ University of Turin, Drug Science and Technology, Turin, Italy; ${ }^{4}$ Fpo Irces Candiolo, Hospital Pharmacy, Candiolo, Italy

10.1136/ejhpharm-2020-eahpconf.64
Background and importance In a comprehensive cancer centre, injectable anticancer drugs are compounded in the pharmacy based preparation unit with the compounding robot APOTECAchemo to guarantee a high level of quality and safety. In 2018, the oncology pharmacy implemented workflow improvements to manage the growing workload due to the centralisation of the activities of a hospital's satellite pharmacy.

Aim and objectives The aim of the study was to analyse the pharmacy workflow and impact of the robotic system on turnaround time to meet increasing pharmacy productivity.

Material and methods Data were collected from the hospital information system and the workflow management software APOTECAmanager, and examined over a 2 year period (2017-2018). The total annual throughput in terms of doses prepared and patients treated were determined. Productivity, number of active ingredients processed, average usage time per day and dosage accuracy (ie, per cent discrepancy between compounded and prescribed dose) were calculated for the robotic system. Medication turnaround time (MTAT) for outpatients, defined as total time from the release of a medication order by the prescriber to administration of the medication to the patient, was measured over 3 months.

Results Overall, the annual doses prepared and patients treated increased by $18 \%$ (from 18574 doses/year in 2017 to 22 754 doses/year in 2018) and by 10\% (from 1421 patients/year in 2017 to 16604 patients/year in 2018), respectively. The robot was used to compound $47 \%$ of the overall doses prepared in 2017 (8677 doses/year) compared with 57\% in 2018 (13 047 doses/year). The average usage time of the robot increased by $58 \%$ (from 2.09 hours/day in 2017 to 5.01 hours/day in 2018), while the number of active ingredients processed rose from 20 in 2017 to 30 in 2018. Overall, $95.0 \%$ of doses prepared with the robot showed an error $<2.0 \%$. The average MTAT for outpatients was lowered by 24.7\% (from 2.8 hours in 2017 to 2.1 hours in 2018).

Conclusion and relevance The study revealed that the oncology pharmacy was able to meet escalating demands of ready to administer anticancer drugs by satellite hospitals, by making more use of the robotic system without increasing pharmacy staff. The pharmacy workflow changes enabled reduced MTAT and better order processing efficacy, thereby providing improved patient care.

\section{REFERENCES AND/OR ACKNOWLEDGEMENTS}

No conflict of interest.

\section{PC-018 MITOMYCIN C STABILITY ACCORDING TO PH AND TEMPERATURE CONDITIONS}

G Miralles Andreu*, I Truyols García, M Pomares Bernabeu, L Soriano Irigaray, L Peral Ballester, A Navarro Ruiz. Hospital General Universitario De Elche, Hospital Pharmacy Department, Elche, Spain

\subsection{6/ejhpharm-2020-eahpconf.65}

Background and importance Mitomycin $\mathrm{C}$ is used in different regimens for the treatment of bladder, anus and lung cancer. According to the data sheet, reconstitution of the vial should be carried out with water for preparation of injectables or with $20 \%$ dextrose. Despite this, sodium chloride solutions are commonly used for its administration. However, it is known that the stability of mitomycin $\mathrm{C}$ molecule is affected by the $\mathrm{pH}$ of the preparation as degradation increases with $\mathrm{pH}$ values $<7$. Sodium chloride solutions have an approximate 